\title{
Epithelioid Trophoblastic Tumour Presenting with Scalp Lesions
}

\author{
Raquel Basto*1 $^{* 1}$ Inês Brandão Rêgo*2 ${ }^{* 2}$ Joana Correia Magalhães ${ }^{1}$, Maria João Sousa ${ }^{1}$, Tatiana Cunha Pereira ${ }^{1}$, Sofia Broco $^{1}$, \\ Isabel Pazos ${ }^{1}$, Teresa Carvalho ${ }^{1}$, Gabriela Sousa ${ }^{1}$ \\ ${ }^{1}$ Medical Oncology Department, Instituto Português de Oncologia de Coimbra, Francisco Gentil, E.P.E., Coimbra, Portugal \\ ${ }^{2}$ Medical Oncology Department, Centro Hospitalar e Universitário de São João, Porto, Portugal \\ ${ }^{*}$ Both authors contributed equally.
}

Received: 14/09/2021

Accepted: 11/10/2021

Published: $25 / 10 / 2021$

How to cite this article: Basto R, Brandão Rêgo I, Correia Magalhães J, Sousa MJ, Cunha Pereira T, Broco S, Pazos I, Carvalho T, Sousa G. Epithelioid trophoblastic tumour presenting with scalp lesions. EJCRIM 2021;8: doi:10.12890/2021_002870.

Conflicts of Interests: The authors declare there are no competing interests.

This article is licensed under a Commons Attribution Non-Commercial 4.0 License

\section{ABSTRACT}

Epithelioid trophoblastic tumour (ETT) is a very rare variant of gestational trophoblastic disease, which arises in reproductive-age women with a prior gestational history. Because of its rarity, its biological behaviour, imaging characteristics and therapeutic schedule have not yet been fully established. Here we describe a rare case of metastatic ETT in a premenopausal woman. A 40-year-old, gravida 3, para 2, Portuguese woman was referred to the dermatology department for multiple skin nodules on the scalp measuring between 1 and $6 \mathrm{~cm}$. A skin biopsy was suggestive of metastatic lesions of low differentiated carcinoma in favour of squamous cell carcinoma. Staging cervicalthoracic-abdominal and pelvic CT showed multiple lesions compatible with metastasis (ganglia, lung and kidneys). Since a CT scan was unable to identify the primary tumour, it was decided to perform a PET-CT scan and to take a biopsy of a vulvar nodule which had been clinically identified as ETT. The patient began the EMA-CO protocol and completed two cycles but with clinical worsening and radiological progression. Although several different chemotherapy regimens are used to treat gestational trophoblastic disease, the optimal treatment is not known given the rarity of this disease and the lack of controlled trials.

\section{LEARNING POINTS}

- Trophoblastic disease is a rare entity with several presentations at diagnosis.

- Presentation with dermatological changes may need differential diagnosis to distinguish it from other types of dermatological disease.

- Quick diagnosis and referral to an appropriate centre are needed.

\section{KEYWORDS}

Trophoblastic, disease, gynecological, cancer

\section{INTRODUCTION}

Epithelioid trophoblastic tumour (ETT) is a very rare type of gestational trophoblastic disease (GTD), which develops in reproductive-age women with a prior gestational history [1]. The different types of malignant GTD include choriocarcinoma (CC), placental site trophoblastic tumour (PSTT), placental site nodule (PSN), epithelioid trophoblastic tumour (ETT) and invasive mole (IM) that may not resolve spontaneously ${ }^{[2-4]}$.

The origin of ETT lies in the intermediate trophoblastic cells of chorion laeve. This type of tumour has some typical features that include nuclear hyperchromasia and pleomorphism, epithelioid nests, solid tumour masses, circumscribed margins, absence of vascularization within the tumour, variable mitosis, an enlarged area of necrosis, absence of chorionic villi, and hyalinization ${ }^{[1]}$. 
Because of its rarity, there are no reported pathognomonic symptoms of ETT, but abnormal vaginal bleeding has been documented as a usual ETT symptom ${ }^{[1,3]}$.

Regarding the location of the tumour, most ETTs develop in the uterus (70\%), specifically in the lower segment; however, the disease may present with an extra-uterine location (lung, small bowel, vagina, fallopian tube, broad ligament and gallbladder) or uterine location with distant metastases. Of the reported cases of ETT with metastasis, the lung was the most common site of extra-uterine ETT (19\%), and metastasis occurred in $25-42.9 \%$ of cases ${ }^{[4]}$.

An accurate diagnosis based on morphological and immunohistological features is very important and essential for appropriate treatment, and allows differentiation between non-neoplastic lesions and neoplastic lesions such as PSTTs and ETTs (both with similar microscopic findings and slow-growing behaviour), potentially locally invasive lesions, or trigger metastases. This is particularly important because of all the GTDs, ETT is the only neoplasm whose curative treatment involves surgery, due to its high chemoresistance ${ }^{[3]}$.

Given the rarity of this entity, its biological behaviour, imaging characteristics and therapeutic schedule are not fully established. Herein, we report a rare case of metastatic ETT in a 40-year-old premenopausal woman.

\section{CASE DESCRIPTION}

A 40-year-old, gravida 3, para 2, Portuguese woman was referred to the dermatology department of our hospital for multiple skin nodules on the scalp measuring between 1 and $6 \mathrm{~cm}$. The first lesion had developed 9 months before referral, was in the right frontal region of the scalp and was about $6 \mathrm{~cm}$ in diameter. It disappeared spontaneously in 8 weeks, but 1 month later a second lesion emerged in the right temporo-occipital region of the scalp (Fig. 1A) with approximately the same size. Numerous nodular lesions then developed on the scalp with the largest in the left parietal region (Fig. 1B). Clinically, they were suggestive of keratoacanthoma (lumps with central necrosis and well-defined borders). However, skin biopsy was suggestive of metastatic lesions of low differentiated carcinoma in favour of squamous cell carcinoma (SCC).

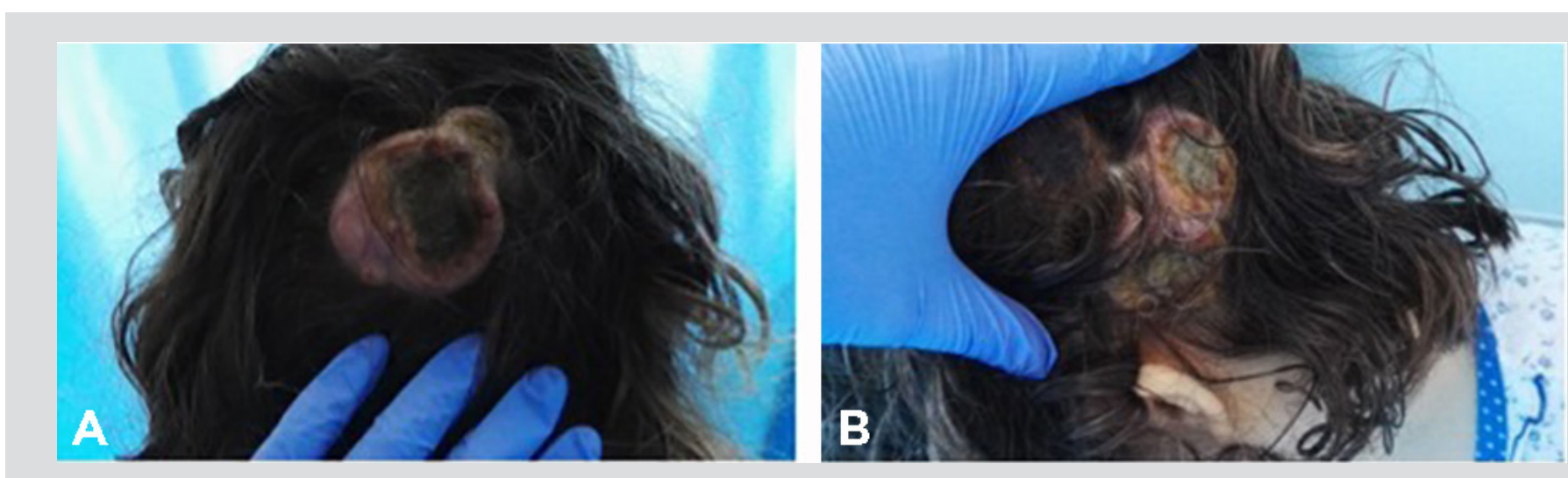

Figure 1. Photographs of the first documented lesions. (A) Lesion on the right temporo-occipital region of the scalp. (B) Lesion on the left parietal region of the scalp

Tumour markers at the time were: serum cancer antigen 125 (Ca 125) 144 U/ml (normal value <35 U/ml), human epididymis protein 4 (HE4) $76,200 \mathrm{pmol} / \mathrm{I}$ (normal value $<76.2 \mathrm{pmol} / \mathrm{l}$ ) and beta-human chorionic gonadotropin ( $\beta$-hCG) $556 \mathrm{mIU} / \mathrm{I}$ (normal value $<5 \mathrm{mIU} / \mathrm{I}$ ).

Staging cervical-thoracic-abdominal and pelvic CT showed laterocervical bilateral lymph nodes, multiple pulmonary nodules, suggestive of secondary lesions (the largest measuring $28 \mathrm{~mm}$ in the upper left lobe) (Fig. 2A), and nodules in both kidneys, also suggestive of metastases (Fig. 2B).

Since CT scanning was unable to identify the primary tumour, it was decided in a multidisciplinary meeting to perform a PET-CT scan for further investigation and to take a biopsy of a vulvar nodule, which had been recently clinically identified, for additional histological information.

The PET-CT scan showed a hypermetabolic lesion in the upper lobe of the left lung (SUVmax: 11.6) measuring $36 \times 22 \mathrm{~mm}$ and suggestive of a high-grade cancer lesion (Fig. 3A), multiple bilateral pulmonary nodules suggestive of metastatic lesions (Fig. 3B), multiple hypermetabolic lesions in both kidneys (Fig. 4A) and splenomegaly (Fig. 4B). 

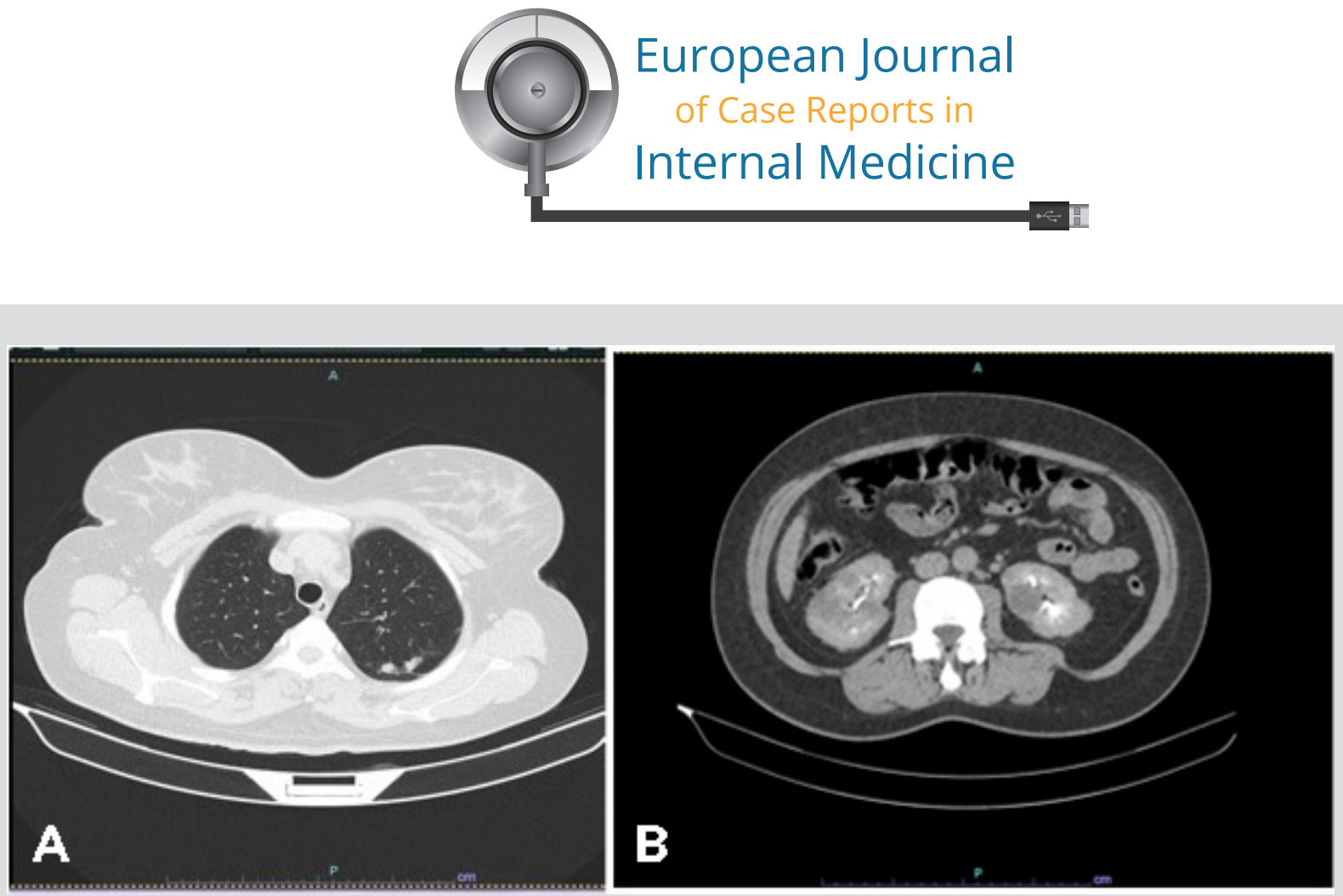

Figure 2.CT images showing nodules in the left upper lobe of the lung $(A)$ and renal nodules (B), compatible with secondary lesions
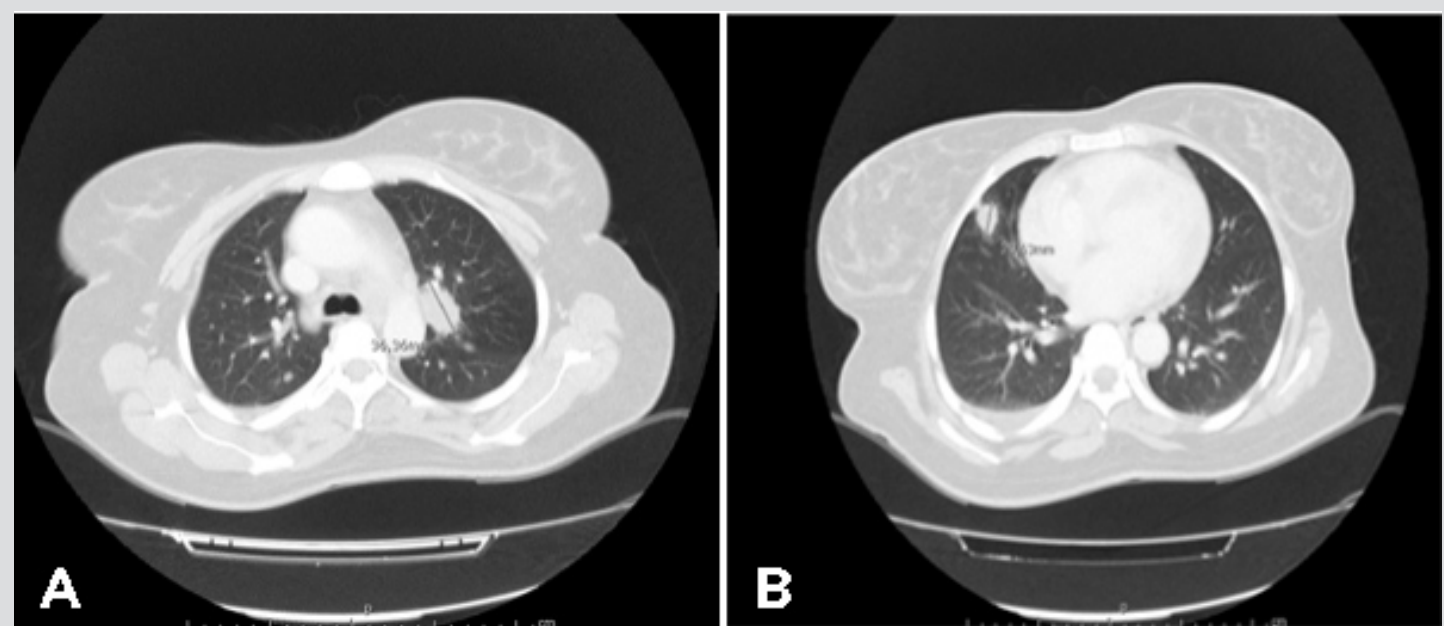

Figure 3. PET-CT images of pulmonary lesions compatible with metastasis. (A) Lesion in the upper lobe of the left lung (SUVmax: 11.6$)$ measuring $36 \times 22 \mathrm{~mm}$. (B) Multiple bilateral pulmonary nodules suggestive of metastatic lesions.
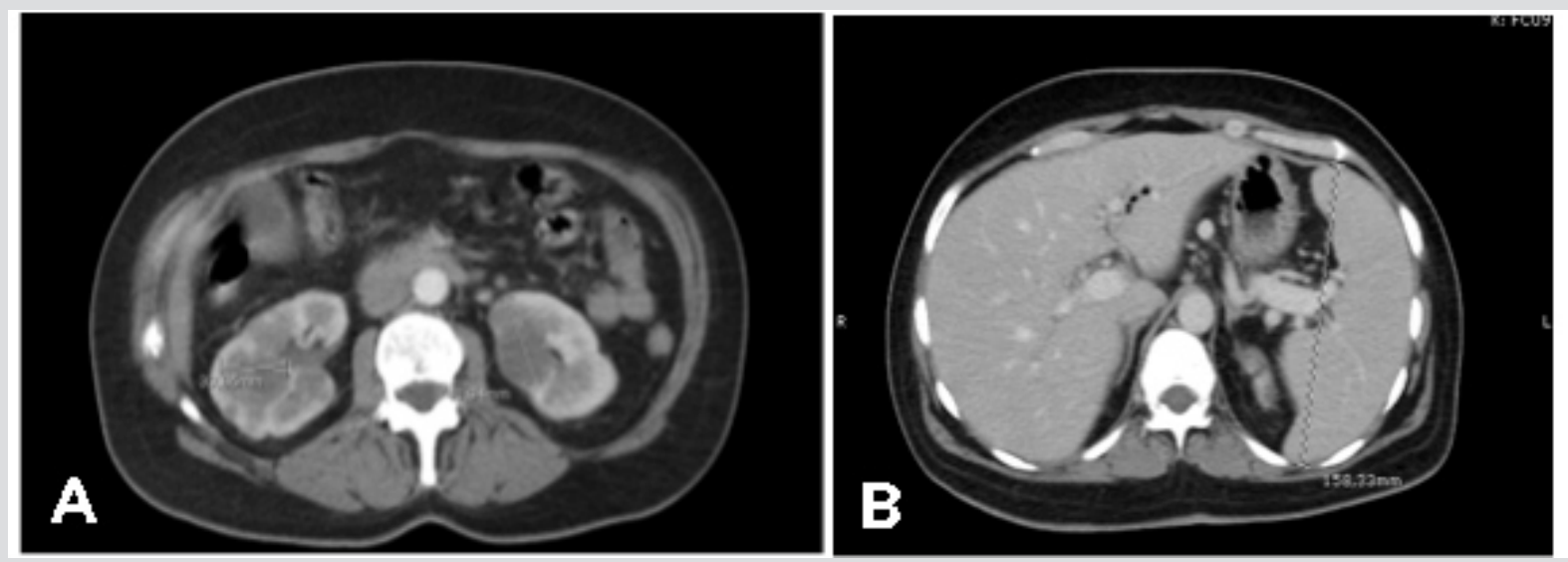

Figure 4. PET-CT images of kidney lesions compatible with metastasis (A) and splenomegaly (B) 
Histology of the biopsy specimen from the left hemi-vulvar lesion was consistent with a metastatic lesion with similar characteristics to skin lesion biopsies previously analysed; however, after complementary anatomopathological studies, the diagnosis of the ETT was made (Fig. 5).

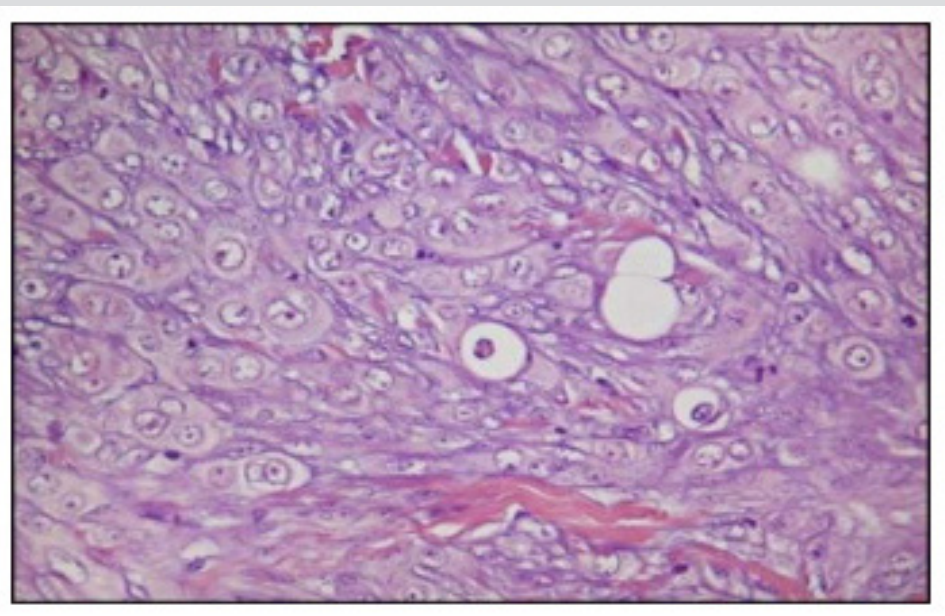

Figure 5. Histological examination revealed atypical epithelioid cells with eosinophilic cytoplasm and large and pleomorphic nuclei (H\&E, 400x)

In July 2017, after discussion at a multidisciplinary meeting, it was decided to start chemotherapy with the multidrug regimen of etoposide, methotrexate, dactinomycin (Act-D), cyclophosphamide and vincristine (EMA-CO: Day 1 - etoposide $100 \mathrm{mg} / \mathrm{m} 2+0.5 \mathrm{mg}$ dactinomycin and $300 \mathrm{mg} / \mathrm{m}^{2}$ methotrexate; Day 2 - etoposide $100 \mathrm{mg} / \mathrm{m}^{2}+0.5 \mathrm{mg}$ dactinomycin and leucovorin $15 \mathrm{mg} q 12 \mathrm{~h}$ for four doses; Day 8 - vincristine $0.8 \mathrm{mg} / \mathrm{m}^{2}$ and cyclophosphamide $600 \mathrm{mg} / \mathrm{m}^{2}$, every 14 days until three cycles after a decrease in $\beta$-hCG tumour marker levels to $<5 \mathrm{mIU} / \mathrm{ml}$ ). After two cycles (last administration in September 2017), the patient presented with clinical deterioration with uncontrolled severe back and left scapula pain with radiation to the neck, holocranial headaches, and visible progression of the scalp skin lesions (Fig. 6).

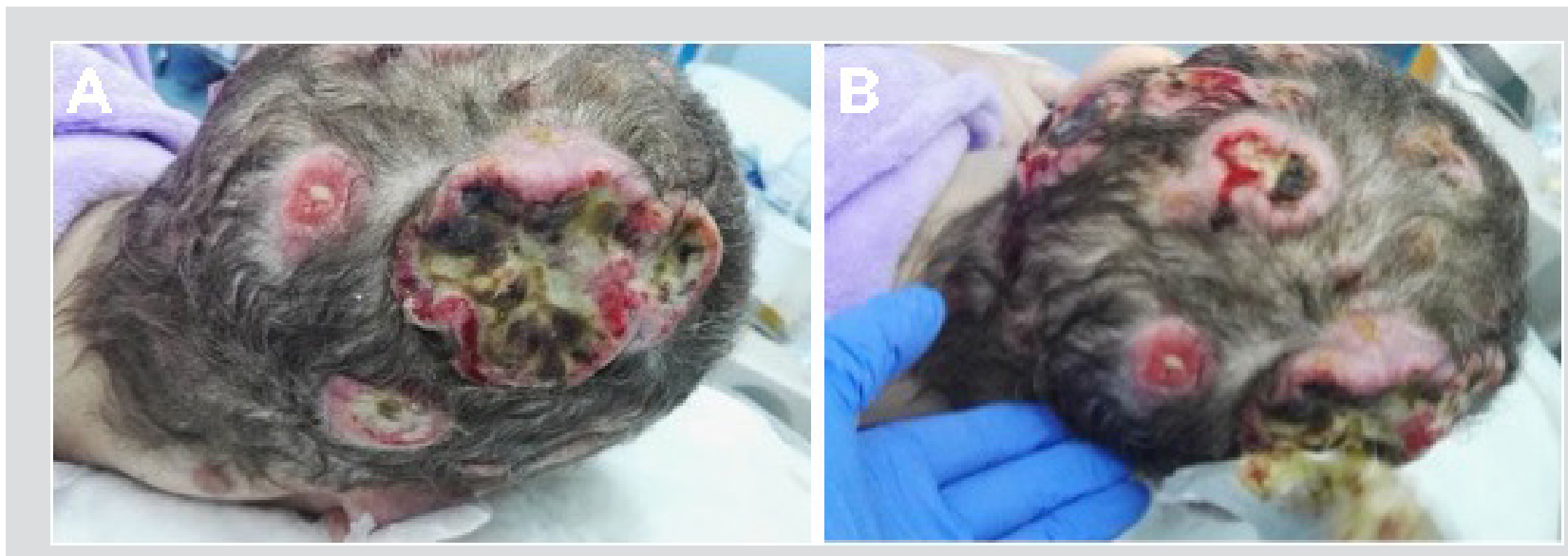

Figure 6. Scalp lesions after two cycles of chemotherapy

The patient was admitted for pain control with an opioid-based multidrug regimen. A cervical-thoracic-abdominal and pelvic CT scan showed increased pulmonary lesions $(40 \mathrm{~mm}$ ) (Fig. 7A), bilateral pleural effusion (Fig. 7B), myocardial lesions (42 mm) and iliac lymph nodes (Fig. 8), and increased kidney lesions (50 mm) (Fig. 9).

While in hospital, the patient developed skin lesion infections, external otitis and a respiratory infection, which all resolved by the time of hospital discharge 1 month later.

A few weeks later, the patient's cervical back pain worsened and was resistant to pain relief. A cervical spine CT scan was performed and showed multiple expansive lesions in the cervical muscular and subcutaneous region with compression of radicular nerves C2-C6 (Fig. 10). It was decided to start antalgic radiotherapy for the two tumoural masses in the lateral cervical region. The patient did not tolerate the bodysteady position necessary for the radiotherapy and it was discontinued a few days later. The patient was maintained under palliative care and died in December 2017. 

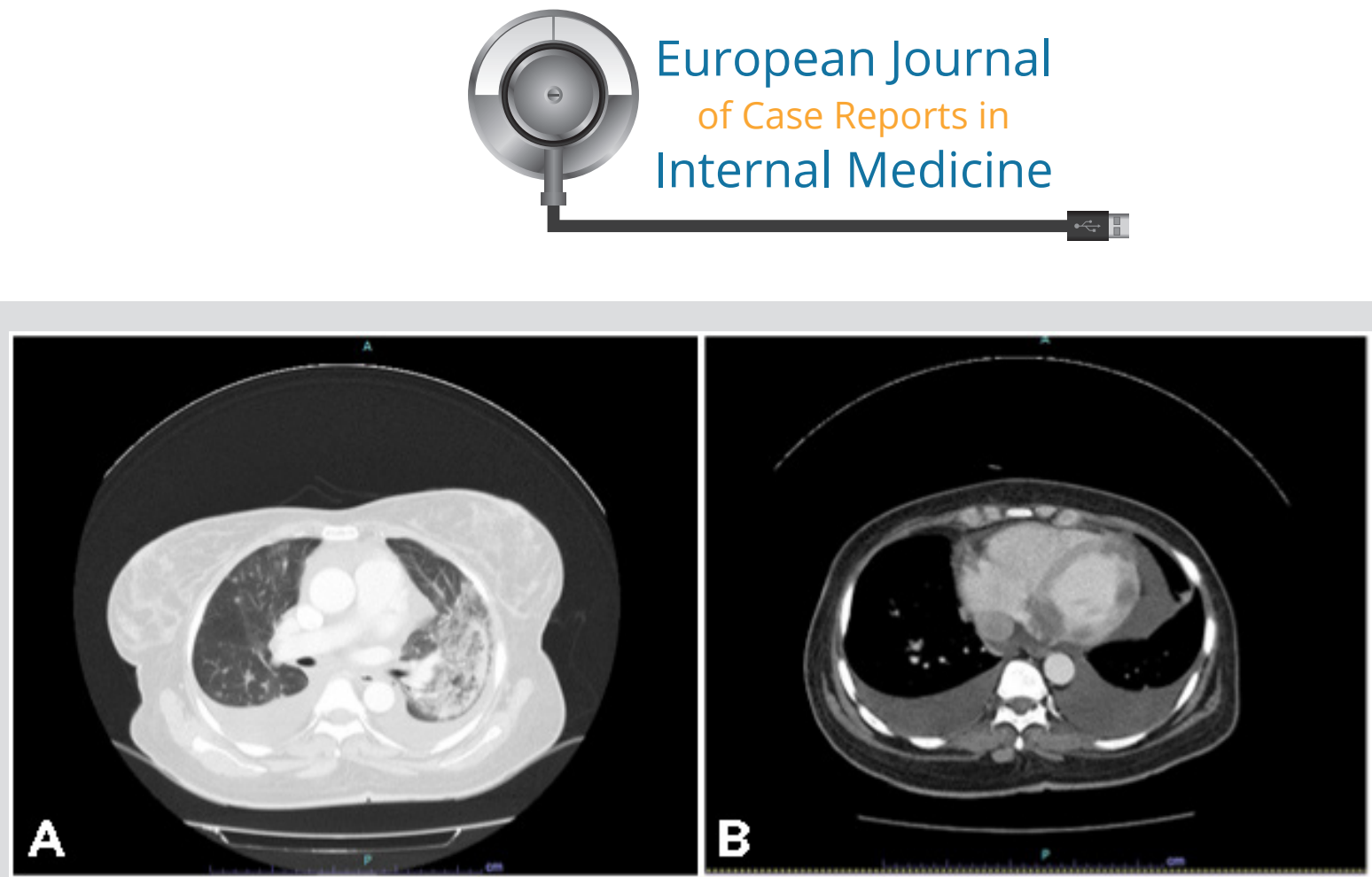

Figure 7. CT images showing increased pulmonary lesion (A) and bilateral pleural effusion (B)

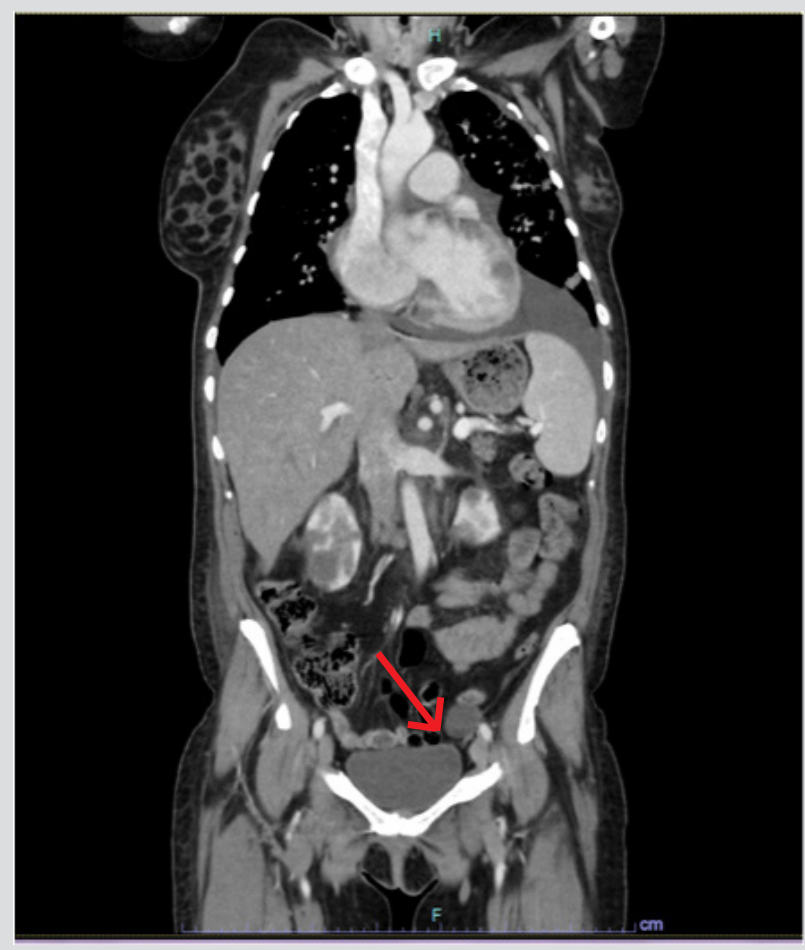

Figure 8. CT images showing myocardial lesions (blue arrow) and iliac lymph nodes (red arrow)

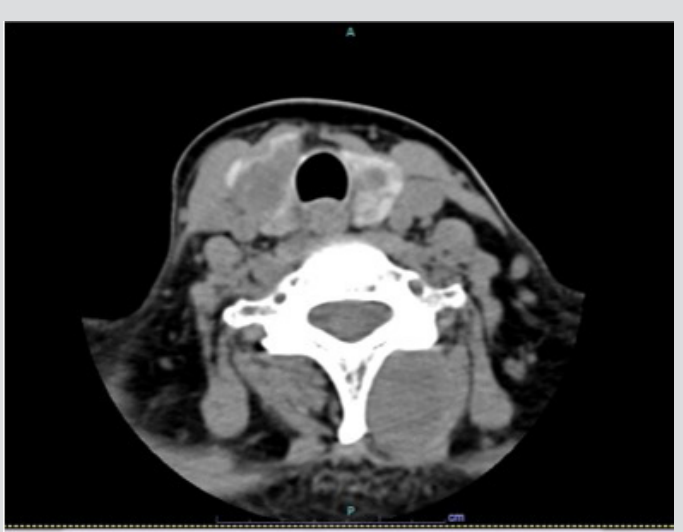

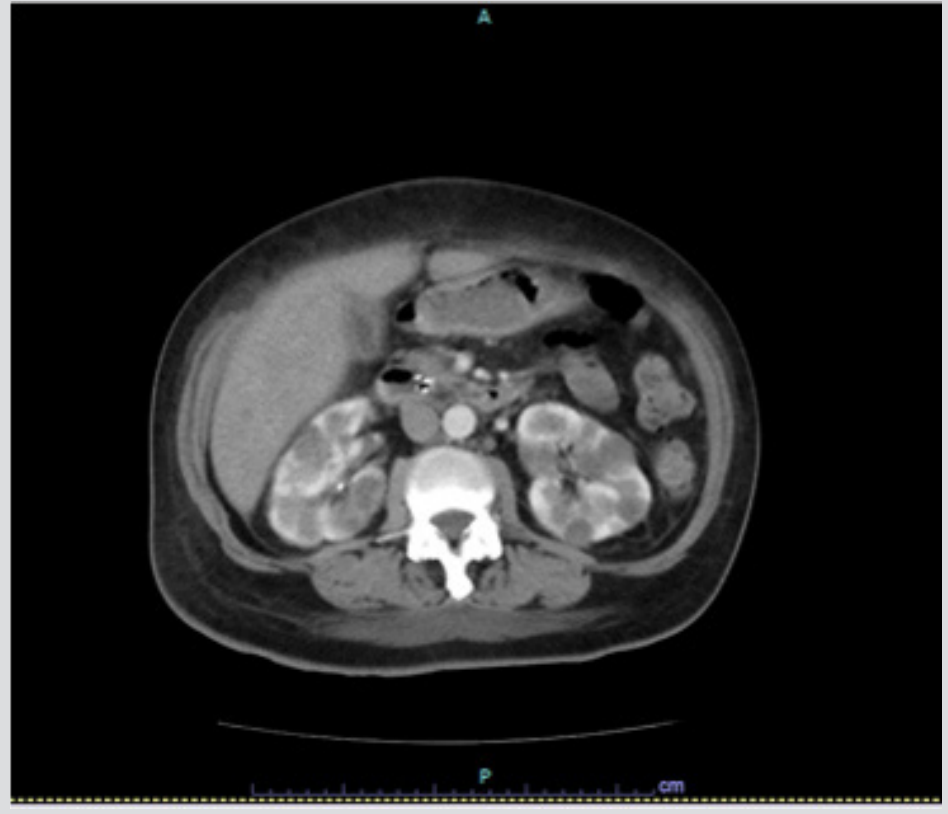

Figure 9. CT images showing increased kidney lesions 


\section{DISCUSSION}

Our case had some peculiarities that contributed to the complexity of the diagnosis. Our patient had plurimetastatic disease, which is an infrequent finding in this type of pathology, with indolent behaviour, high-volume extrauterine disease and an atypical clinical manifestation with cranial skin lumps ${ }^{[3]}$.

As stated earlier, due to its rarity and absence of pathognomonic signs and symptoms, the diagnosis of ETT can be easily missed. Therefore, imaging exams, such as CT or MRI, are urgent and essential ${ }^{[3]}$. In our case, PET-CT revealed a hypermetabolic lesion in the upper lobe of the left lung (SUVmax: 11.6) measuring $25 \times 22 \mathrm{~mm}$ and suggestive of a high-grade cancer lesion, multiple bilateral pulmonary nodules suggestive of metastatic lesions, hyperbolic adenopathy in the aortopulmonary window measuring $23 \times 12 \mathrm{~mm}$ (SUVmax: 11.2) suggestive of ganglia metastasis, multiple locations of hypermetabolic activity in the left ventricle (SUVmax: 6.7), suggestive of secondary lesions, and also multiple hypermetabolic lesions in both kidneys and pancreas.

The role of tumour markers is also important in this pathology. In this specific case, $\beta$-hCG was important in distinguishing between ETT and other GTDs, such as CC and PSTT. In ETT, in contrast to CC, serum $\beta$-hCG is usually elevated but to a lower level ( $\beta$-hCG $<2500 \mathrm{mIU} / \mathrm{ml})^{[2-4]}$. At the microscopic level, some features favour the diagnosis of ETT, as stated previously. ETT is a neoplasm formed by intermediate trophoblastic cells, cells that are also present in other GTDs (PSTT, PSN). The differential diagnosis to distinguish between these three pathologies is based on immunohistochemical characteristics: in PSN there is no necrosis, the site nodules are usually smaller, and Ki67 is low (vs. ETT); as PSTT and ETT share some features (cellular population, fibrinoid tissue and absence of chorionic villi), the immunohistochemical characteristics of ETT determine the differential diagnosis with PSTT ${ }^{[1-3]}$.

Analysis of the expression of immunohistochemical markers is important and useful for directing diagnosis. Essential markers in this entity are $\mathrm{p} 63, \beta$-hCG, hP, PLAP, E-cadherin, inhibin alpha antibody, cytokeratins AE1/AE3, prolyl-4-hydroxylase epidermal growth factor receptor, and the Ki67 index. Regarding the differential diagnosis, p63 expression is absent in PSTT (vs. 45-65\% in ETT); Ki67 is <10\% in PSN, 15-25\% in PSTT and $>50 \%$ in SCC (vs. 10-25\% in ETT); and cytokeratin-18 and inhibin alpha antibody are absent in SCC (vs. ETT) ${ }^{[1-3]}$.

\section{CONCLUSION}

Of the gestational trophoblastic neoplasias, ETT is a very rare tumour. The clinical presentation varies greatly between patients, with some having amenorrhoea and a negative $\beta$-hCG. Diagnostic accuracy is the gold standard in guiding this pathology, since immunohistological tests are the only way to distinguish between some GTD variants (ETT vs. choriocarcinoma).

Several cytostatic regimens used are used for treatment, but due to the rarity of the pathology and the scarcity of controlled clinical trials, it is still not possible to determine the best option.

\section{REFERENCES}

1. Stănculescu RV, Bauşic V, Vlădescu TC, Vasilescu F, Brătilă E. Epithelioid trophoblastic tumor: a case report and literature review. Rom J Morphol Embryol 2016;57(4):13651370.

2. Bruce S, Sorosky J. Gestational trophoblastic disease. StatPearls [Internet] 2020.

3. Horowitz NS, Goldstein DP, Berkowitz RS. Placental site trophoblastic tumors and epithelioid trophoblastic tumors: biology, natural history, and treatment modalities. Gynecol Oncol 2017;144(1):208-214.

4. Zhang X, Lü W, Lü B. Epithelioid trophoblastic tumor: an outcome-based literature review of 78 reported cases. Int J Gynecol Cancer 2013;23(7):1334-1338. 